\title{
MÉTODOS DE PREVENÇÃO E REAQUECIMENTO DO PACIENTE PARA O PERIOPERATÓRIO
}

\author{
METHODS OF PREVENTION AND REWARMING OF THE PATIENT IN PERIOPERATIVE
}

MÉTODOS DE PREVENCIÓN Y DE REAQUECIMENTO DEL PACIENTE EN PERIOPERATORIO

\section{Vanessa de Brito Poveda ${ }^{1}$, Marister Piccoli², Cristina Maria Galvão ${ }^{3}$ Namie Okino Sawada ${ }^{3}$}

RESUMO: Este estudo teve como objetivo investigar as evidências disponíveis na literatura sobre os métodos de aquecimento utilizados para prevenção e tratamento da hipotermia no perioperatório. Para tanto, realizou-se uma revisão da literatura nacional e internacional por meio dos indexadores Medline, em busca de artigos, teses ou dissertações publicados na íntegra no período compreendido entre 1995 a 2005, utilizando-se as palavras chaves hipotermia, assistência perioperatória e reaquecimento totalizando 20 artigos. De acordo com os estudos analisados concluímos que o melhor método de aquecimento para os pacientes no período perioperatório, especialmente no pós-operatório imediato, é o cobertor de ar aquecido, outro método comparável seria o resistive heating. Métodos mais conhecidos e utilizados em nossa realidade não demonstraram efetividade comparável aos métodos citados acima, como o cobertor de algodão e o colchão de água aquecida, vale lembrar também, os riscos associados ao colchão de água aquecida como queimaduras, por exemplo. Assim, apesar do custo elevado destas tecnologias devemos ressaltar a importância da prevenção da hipotermia como uma medida efetiva na redução de custos, além dos efeitos deletérios causados por ela na recuperação pós-operatória destes pacientes.

PALAVRAS-CHAVE: Hipotermia; Reaquecimento; Assistência Perioperátória.

ABSTRACT: This study aimed to investigate the available evidences on literature about rewarming methods used in perioperative hypothermia prevention and treatment in perioperative time. To develop this we search for fully national or international published papers, thesis or dissertations indexed in Medline, from 1995 to 2005, by the key words hypothermia, perioperative care and rewarming. It was found 20 articles. These studies showed the better rewarming methods to patients in perioperative, especially immediate post operative is the forced air warming; other comparable was the resistive heating. Other methods utilized in our country, like cotton blanket and heating water mattress, are not efficient and can burn the patients, for example. Thus, although the high cost of these technologies we have to salient the hypothermia prevention importance as an effective way on coasts reduction, and deleterious effects provoked for it on post operative rehabilitation of these patients.
KEYWORDS: Hypothermia; Rewarming; Perioperative Care.

RESUMEN: El estudio tuvo como objetivo investigar la evidencia disponible en literatura sobre los métodos de reaquecimento usados en la prevención y el tratamiento perioperatorio de la hipotermia. Para realizar esto, buscamos el literatura nacional y internacional en Medline, entre 1995 y 2005, con las palabras claves, hipotermia, atención perioperatorio y recalentamiento. Fueron encontrados 20 artículos. Estos estudios demostraron los métodos de reaquecimento mejores a los pacientes en perioperatorio, especialmente inmediato el calentamiento forzado del aire; el otro comparable fuera la calefacción resistente. Otros métodos utilizados en nuestro país, como manta de algodón y el colchón con agua calentada, no son eficientes y pueden provocar quemaduras, por ejemplo. Así, aunque se tiene alto costo de estas tecnologías nosotros reforzamos la importancia de prevención de la hipotermia como manera eficaz en la reducción de los costos financieros, y efectos deletéreos provocados para esto en la recuperación post-operativa de estos pacientes.

PALABRAS CLAVE: Hipotermia; Recalentamiento; Atención Perioperativa.

\footnotetext{
Enfermeira. Doutoranda do Programa de Doutorado em Enfermagem Fundamental da Escola de Enfermagem de Ribeirão Preto - USP. Docente das Faculdades Integradas Teresa D'Ávila (FATEA) São Paulo.

Enfermeira. Doutoranda do Programa de Doutorado em Enfermagem Fundamental da Escola de Enfermagem de Ribeirão Preto - USP. Docente do Colegiado de Enfermagem da Universidade Estadual do Oeste do Paraná.

${ }^{3}$ Enfermeira, Doutora em Enfermagem. Professor Associado da Escola de Enfermagem de Ribeirão Preto, da Universidade de São Paulo, Centro Colaborador da OMS para o desenvolvimento da pesquisa em enfermagem. Ribeirão Preto, São Paulo. E-mail: crisqalv@eerp.usp.br
} 
POVEDA, Vanessa de Brito; PICCOLI, Marister; GALVÃO, Cristinha Maria; SAWADA, Namie Okino. MÉTODOS DE PREVENÇÃo E REAQUECIMENTO DO PACIENTE PARA O PERIOPERATÓRIO. Revista Eletrônica de Enfermagem, v. $07, \mathrm{n} .03, \mathrm{p} .266$ - $272,2005$. Disponível em http://www.fen.ufg.br/Revista/revista7_3/original 02.htm

\section{INTRODUÇÃO}

BORMS et al (1994) estimam que a hipotermia perioperatória atinja de 50 a $90 \%$ dos pacientes, tornando este problema bastante freqüente em sala operatória.

As salas cirúrgicas devem contar com sistemas de ar condicionado de maneira que a temperatura dentro delas, mantenha a equipe confortável (20 a $23^{\circ} \mathrm{C}$ ), a qual utiliza os componentes da paramentação cirúrgica. O paciente freqüentemente está desnudo, além de outras situações, como o uso de solução de anti-sepsia fria, exposição de grande área de pele, abertura das cavidades torácica ou abdominal, aumentando a área de perda de calor, infusão de soluções frias, inalação de gazes anestésicos frios; além disso, salientamos os efeitos dos anestésicos que são potentes vasodilatadores (GARANHANI et al,1990; PETERSON, 2003; LESLIE \& SESSLER, 2003).

Vale lembrar, que o corpo perde calor através de quatro mecanismos, conhecidos como: radiação, onde o organismo perde calor para o ambiente mais frio do que seu corpo, por meio de ondas eletromagnéticas, corresponde a $60 \%$ de sua eliminação térmica total; condução corresponde à perda calórica mínima (3\%), onde se transfere a energia térmica pelo contato direto com as superfícies; convecção, ocorre remoção do calor do corpo por correntes de ar, correspondendo a aproximadamente $12 \%$ da perda total e por evaporação, quando se perde água por evaporação da superfície corporal (GUYTON \& HALL, 2002).

Todavia, quando o organismo é resfriado ele dispõe de mecanismos que atuam no sentido contrário, tentando manter a normotermia, são eles: a vasoconstricção da pele, piloereção, abolição da sudorese, aumento da produção de calor e calafrios (GUYTON \& HALL, 2002).

A normotermia consiste na temperatura corporal entre $36^{\circ} \mathrm{C}$ e $38^{\circ} \mathrm{C}$ e hipotermia como a temperatura corporal menor que $36^{\circ} \mathrm{C}$; com base na temperatura central, a hipotermia pode ser considerada leve (32 a $\left.35^{\circ} \mathrm{C}\right)$, média ou moderada $\left(28\right.$ a $\left.32^{\circ} \mathrm{C}\right)$ e grave ou severa (menor que $28^{\circ} \mathrm{C}$ ) (MANNING \& STOLLERMAN, 1993).

Pode ainda ser classificada como primária, quando está relacionada a alguma falha do sistema nervoso central; secundária quando está associada a doenças de diversas naturezas, infecção grave, uso de drogas, má nutrição e acidental, ou seja, aquela que ocorre por exposição a baixas temperaturas, portanto, a que ocorre com o paciente no intra-operatório (MANNING \& STOLLERMAN, 1993).

Segundo STEVENS et al (2000) a hipotermia afeta o paciente cirúrgico de diversas maneiras, causando efeitos em diversos sistemas: pulmonar: desvio a esquerda da curva de oxihemoglobina, mudanças na função pulmonar, diminuição da ventilação, aumento da necessidade de oxigênio; cardiovascular: diminuição do volume sanguíneo efetivo, arritmias ventriculares, depressão miocárdica e isquemia; altera a função plaquetária, levando ao aumento do sangramento; neurológico: depressão cerebral generalizada, disfunção do hipotálamo; metabólico: diminuição na taxa de metabolismo basal, prolongamento do efeito das medicações, alteração da eliminação hepática de substâncias e medicações; gastrointestinal: diminuição da motilidade intestinal; geral: aumento do número de infecção no corte cirúrgico.

DOUFAS (2003) ao revisar a literatura afirma que o acréscimo de $1,9^{\circ} \mathrm{C}$ na hipotermia central triplica a incidência de infecção no corte cirúrgico, bem como, o aumento da duração da hospitalização em até 20\%, afeta negativamente a ação dos anticorpos e defesa imune mediada por células, tanto quanto, a disponibilidade de oxigênio para tecidos periféricos. Além destes aspectos, a hipotermia leve também altera a cinética e ação de vários agentes anestésicos, estando relacionada a desconforto térmico e demora na recuperação pós-anestésica.

Em relação às drogas utilizadas na anestesia, muitas têm seus efeitos alterados em virtude da hipotermia, em geral a ação dos relaxantes musculares, como o vencurônio, tem seu efeito aumentado, da mesma forma com os anestésicos voláteis e intravenosos (DOUFAS, 2003).

LESLIE \& SESSLER (2003) além dos efeitos já citados anteriormente, ressaltam alguns aspectos positivos da hipotermia, como a proteção ao cérebro de isquemia e a preservação do músculo miocárdico.

$\mathrm{Na}$ prevenção dos efeitos deletérios relacionados à hipotermia, estudiosos têm procurado investigar medidas efetivas na prevenção e correção da hipotermia no período perioperatório.

Neste sentido, a AMERICAN SOCIETY OF PERIANESTHESIA NURSES (2001) desenvolveu um guia clínico para a prevenção da hipotermia no período perioperatório. Sugere que no período pré-operatório identifiquem-se os fatores de risco para hipotermia, como: extremos de idade, sexo feminino, temperatura da sala, duração e tipo do procedimento cirúrgico, caquexia, doenças pré-existentes, perda significante de fluidos, uso de soluções frias, anestesia geral ou regional. Neste período, a temperatura do paciente deve ser verificada na admissão, o enfermeiro deve perguntar ao paciente se sente frio e observar sinais e sintomas de hipotermia (tremores, piloereção, e extremidades frias). A partir daí, intervenções devem ser implementadas no sentido de manter o indivíduo normotérmico (por exemplo, uso de cobertor de algodão, meias dentre outras).

No intra-operatório, o enfermeiro necessita avaliar novamente a temperatura do paciente $\mathrm{e}$ monitorá-la durante o transcorrer do procedimento anestésico-cirúrgico. Recomenda-se a implementação de medidas de aquecimento como o uso de cobertor, meias, capuz, exposição limitada da pele, utilização de aparelho de aquecimento artificial, administração de 
POVEDA, Vanessa de Brito; PICCOLI, Marister; GALVÃO, Cristinha Maria; SAWADA, Namie Okino. MÉTODOS DE PREVENÇÃo E REAQUECIMENTO DO PACIENTE PARA O PERIOPERATÓRIO. Revista Eletrônica de Enfermagem, v. $07, \mathrm{n} .03, \mathrm{p} .266$ - $272,2005$. Disponível em http://www.fen.ufg.br/Revista/revista7_3/original 02.htm

soluções intravenosa e de irrigação aquecidas, umidificação e aquecimento dos gases anestésicos.

$\mathrm{Na}$ sala de recuperação pós-anestésica a temperatura do paciente hipotérmico deve ser verificada a cada 30 minutos, até atingir a normotermia, caso o paciente esteja normotérmico a temperatura deverá ser mensurada na alta e por solicitação médica.

No caso de pacientes normotérmicos, devem ser implementadas medidas preventivas como o uso de cobertores, meias, capuz, limitar a exposição da pele e infusão de líquidos aquecidos, manter a temperatura da sala entre 20 e $24^{\circ} \mathrm{C}$, aferir a temperatura a cada 30 minutos, observar sinais e sintomas de hipotermia.

No caso de pacientes hipotérmicos recomendase utilizar aparelhos de ar aquecido (manta ou cobertor), cobertor de algodão, meias, capuz, exposição limitada da pele, aumentar a temperatura ambiente, infundir soluções aquecidas, umidificação e aquecimento do oxigênio, e monitorar a temperatura do paciente a cada 30 minutos, até o conforto térmico.

As pesquisas buscam descrever quais são os melhores métodos para aquecimento do paciente no período perioperatório, em relação a este aspecto, ressaltamos o estudo de GARANHANI et al. (1990), em que os autores analisaram 30 pacientes submetidos a cirurgias endo ou ecto cavitárias e exames rádio-diagnósticos que apresentaram hipotermia na chegada ao Serviço de Recuperação Pós-Anestésica (SRPA), avaliando a eficácia da utilização do cobertor de lã acrílica, do cobertor elétrico e do aquecedor elétrico.

Concluíram que o cobertor de lã, método freqüentemente utilizado nos hospitais, foi menos eficaz em relação aos outros métodos investigados, sendo que o aquecedor elétrico apresentou os resultados mais eficientes.

TRAMONTINI (2000) em sua dissertação de mestrado investigou a eficácia de duas intervenções de enfermagem no intra-operatório: aquecer as áreas adjacentes ao sítio cirúrgico, como cabeça, dorso, membros superiores e inferiores $(n=27)$ ou aquecer 0 dorso $(n=27)$, concluiu que, em qualquer um dos métodos não houve melhora no controle da perda de calor corporal.

STEVENS et al (2000) compararam dois métodos para o aquecimento dos pacientes com hipotermia suave à moderada no pós-operatório imediato, utilizando um aparelho de ar aquecido artificialmente e cobertores aquecidos, não encontrando diferenças no tempo de reaquecimento ou ganho de calor entre os grupos.

Verificamos que existe uma grande divergência em relação aos melhores métodos de aquecimento a serem utilizados no período perioperatório, embora pareça claro os efeitos deletérios ocasionados pela exposição à hipotermia nos pacientes submetidos ao procedimento anestésico-cirúrgico, para elucidarmos esta questão propomos o presente estudo.

\section{REFERENCIAL TEÓRICO}

\section{A prática Baseada em Evidências}

A prática baseada em evidências (PBE) teve origem nos estudos de Archie Cochrane, epidemiologista britânico. O desenvolvimento desta abordagem ocorreu paralelamente ao avanço dos meios de informações, intensificando o acesso aos resultados das pesquisas (ESTABROOKS, 1998; HAMER,1999).

Conforme relata GALVÃO et al (2004) a PBE está sendo discutida principalmente no Canadá, Reino Unido e Estados Unidos da América. Em nosso país, essa abordagem desenvolveu-se primeiramente na medicina em Universidades do estado de São Paulo, Rio de Janeiro e Rio Grande do sul, sendo ainda um movimento a ser construído na enfermagem.

A PBE "é uma abordagem que possibilita a melhoria da qualidade da assistência de enfermagem prestada ao cliente. Essa abordagem envolve a definição de um problema, a busca e avaliação crítica das evidências disponíveis (pesquisas), implementação das evidências na prática e avaliação dos resultados obtidos. A competência clínica do enfermeiro e as preferências do cliente são aspectos incorporados também nesta abordagem, para a tomada de decisão sobre a assistência à saúde" (GALVÃO, 2002).

GALVÃO (2002) relata que na PBE existem recursos que proporcionam a incorporação das evidências disponíveis na prática, destacamos: a revisão sistemática, onde os resultados das pesquisas são coletados, categorizados, avaliados e sintetizados e as diretrizes para a prática clínica que são documentos desenvolvidos sistematicamente para auxiliar os profissionais de saúde e pacientes na tomada de decisões sobre as condições de saúde.

A revisão sistemática na enfermagem pode identificar os efeitos benéficos e nocivos de diferentes intervenções da prática assistencial assim como, estabelecer lacunas do conhecimento e identificar quais as áreas que necessitam de pesquisas com implicações para a assistência de enfermagem prestada ao paciente (MOLONEY \& MAGGS, 1999).

A revisão sistemática pode minimizar algumas barreiras para a utilização de resultados de pesquisas na prática assistencial, devido à falta de tempo do enfermeiro, falhas na busca de pesquisas e deficiência de habilidades para avaliar e sintetizar as pesquisas encontradas; assim, consiste na solução de dificuldades encontradas na prática proporcionando a síntese do conhecimento já produzido oriundo de resultados de pesquisas (CILISKA et al., 2001).

\section{A Enfermagem Perioperatória Baseada em Evidências}

Entendemos que a complexidade da enfermagem perioperatória demonstra a importância 
POVEDA, Vanessa de Brito; PICCOLI, Marister; GALVÃO, Cristinha Maria; SAWADA, Namie Okino. MÉTODOS DE PREVENÇÃo E REAQUECIMENTO DO PACIENTE PARA O PERIOPERATÓRIO. Revista Eletrônica de Enfermagem, v. $07, \mathrm{n} .03, \mathrm{p} .266$ - $272,2005$. Disponível em http://www.fen.ufg.br/Revista/revista7_3/original 02.htm

do conhecimento científico para o seu desenvolvimento, assim, a PBE "é uma abordagem que incentiva o enfermeiro a buscar conhecimento científico, por meio do desenvolvimento de pesquisas ou aplicação de resultados de pesquisa encontrados na literatura, à sua prática profissional" (GALVÃO, 2002).

BEYEA apud GALVÃO (2002) coloca que o enfermeiro perioperatório tem uma posição de liderança no contexto da implementação da prática baseada em evidências sendo que este atua constantemente com a equipe cirúrgica e surgem oportunidade na identificação de problemas clínicos potenciais relevantes, uma vez que na enfermagem perioperatória existem práticas que podem ser avaliadas considerando que ao longo vem sendo reproduzidas sem o questionamento se a origem dessas práticas são baseadas em evidências ou na opinião de profissionais.

GALVÃO (2002) entende que a prática baseada em evidências poderá estar contribuindo com a mudança da enfermagem considerando que esta ainda está enraizada em tradição, rituais e tarefas para uma prática reflexiva tendo como base o conhecimento científico, mais especificamente na enfermagem perioperatória, possibilitando o planejamento e implementação de intervenções que atendam as necessidades do paciente cirúrgico e de seus familiares.

\section{OBJETIVO}

Buscar e avaliar as evidências disponíveis na literatura sobre os métodos de aquecimento utilizados para prevenção e tratamento da hipotermia no perioperatório.

\section{MÉTODOLOGIA}

\section{Revisão da Literatura}

O objetivo geral da revisão da literatura é reunir conhecimentos sobre um determinado assunto entendendo que a utilização desse conhecimento poderá ser aplicado de diversas formas. Em nosso estudo esta revisão teve como propósito "orientação sobre o que é e o que não é conhecido em uma área de investigação, para confirmar qual a pesquisa que pode trazer melhor contribuição para o conhecimento" (POLIT et al, p.125, 2004).

Conforme relatam as autoras acima citadas à capacidade de localizar e identificar a literatura é uma habilidade necessária e que exige habilidades de adaptação com o uso crescente da internet uma vez que continuamente estão sendo introduzidos métodos mais sofisticados para a procura da literatura.

POLIT et al, (2004) comentam também que os recursos impressos estão sendo substituídos pelas bases de dados eletrônicos, mas que a sua disponibilidade não pode ser ignorada, considerando que em locais menores muitas vezes não existe a disponibilidade de recursos para uma busca eletrônica, e também que muitas vezes existe a necessidade da consulta para verificação da literatura mais antiga sobre um determinado assunto e dependo dos critérios e objetivo do estudo.

As autoras relatam que a relevância das referências pode ser julgada rapidamente por meio da leitura do resumo ou da introdução, informam também que se deve confiar principalmente em fontes primárias que são descrições de estudos preparados por alguém que não é o pesquisador original. Os artigos de fontes secundárias são descrições de estudos preparados por alguém que não é 0 pesquisador original, consideram também que os artigos de revisão são fontes secundárias e no caso de serem recentes são locais bons para se iniciar uma busca da literatura uma vez que resumem o que é conhecido e as referências bibliográficas são úteis.

Para alguns assuntos, POLIT et al (2004) orientam que a importância da inclusão na revisão às referências da literatura conceitual, que são relativas a um modelo conceitual ou teoria. $\mathrm{Na}$ literatura conceitual a fonte primária é considerada a descrição da teoria por quem a redigiu e a fonte secundária a crítica ou a discussão da teoria.

As autoras consideram também que outros dois tipos de referências podem ser encontrados através da busca da literatura: os artigos opinativos e os artigos que descrevem casos ou impressões clínicas e colocam que estes que não são de pesquisa podem servir para ampliar o conhecimento sobre o problema em estudo, ilustrar ou demonstrar a necessidade de pesquisas, elas colocam que podem ter participação na formulação das idéias, mas com utilidade limitada em decorrência da subjetividade, ou seja, o estágio do conhecimento sobre o problema de pesquisa.

\section{Formulação da questão para revisão}

Quais são os métodos de aquecimento eficazes utilizados no período perioperatório para a prevenção/tratamento de hipotermia no paciente adulto?

\section{Busca de Estudos}

Realizou-se uma revisão da literatura nacional e internacional por meio da base de dados indexados Medline, em busca de artigos, teses ou dissertações publicados na íntegra no período compreendido entre 1995 a 2005, utilizando-se para tanto as palavras chaves: Hipotermia + perioperatório + reaquecimento = 20 artigos. O acesso ao banco de dados ocorreu em 28 de abril de 2005.

\section{Seleção dos Estudos}

Utilizamos como critérios de inclusão: palavraschaves: hipotermia + perioperatório + reaquecimento; artigos publicados em inglês, português e espanhol; publicados nos últimos 10 anos; de acordo com o 
POVEDA, Vanessa de Brito; PICCOLI, Marister; GALVÃO, Cristinha Maria; SAWADA, Namie Okino. MÉTODOS DE PREVENÇÃo E REAQUECIMENTO DO PACIENTE PARA O PERIOPERATÓRIO. Revista Eletrônica de Enfermagem, v. $07, \mathrm{n} .03, \mathrm{p} .266$ - $272,2005$. Disponível em http://www.fen.ufg.br/Revista/revista7_3/original 02.htm

objetivo proposto; sem distinção de nível de evidência; procedimento cirúrgico realizado com paciente adulto, humano. Os artigos foram selecionados por meio do título e leitura dos resumos, totalizando desta forma 3 artigos concordantes com nossos objetivos e com os critérios de seleção propostos. Foram excluídos 2 artigos em alemão; 4 artigos em japonês; 8 artigos não estavam de acordo com nossos critérios de inclusão e 3 artigos não foram localizados.

\section{Avaliação dos Estudos}

Iniciamos a leitura na íntegra das publicações encontradas, utilizando para maior uniformidade na análise dos dados, o instrumento elaborado e validado no estudo de URSI (2005), assim, classificamos os estudos de acordo com STETLER et al (1998) de acordo com os níveis de evidência, além de verificarmos a metodologia empregada e sua concordância com os resultados encontrados. Os resultados são apresentados na seqüência em tabelas adaptadas de URSI (2005) e de forma descritiva.

\section{APRESENTAÇÃO E DISCUSSÃO DOS RESULTADOS}

NG, S.F. et al. A comparative study of three warming interventions to determine the most effective in maintaining perioperative normothermia. Anesthesia \& Analgesia, v.96, n.1, p. 171-6, jan. 2003.

\begin{tabular}{|c|c|c|c|}
\hline $\begin{array}{l}\text { Detalhamento } \\
\text { Metodológico }\end{array}$ & Intervenção Estudada & Resultados & $\begin{array}{l}\text { Recomendações/ } \\
\text { Conclusões }\end{array}$ \\
\hline $\begin{array}{l}\begin{array}{l}\text { Estudo } \\
\text { randomizado, }\end{array} \text { prospectivo, } \\
\text { controlado, } \\
2000 \text { a outubro de } 2000 \text {. } \\
300 \text { pacientes submetidos } \\
\text { à prótese total de joelho } \\
\text { unilateral, igualmente } \\
\text { randomizados em três } \\
\text { grupos por meio do método } \\
\text { do envelope selado. }\end{array}$ & $\begin{array}{l}\text { Controle - dois cobertores } \\
\text { de algodão } \\
\text { GI - cobertor reflexivo } \\
\text { GII - cobertor de ar } \\
\text { aquecido artificialmente }\end{array}$ & $\begin{array}{l}\text { O grupo experimental } 2 \\
\text { que recebeu o método do } \\
\text { cobertor de ar aquecido } \\
\text { apresentou temperatura } \\
\text { mais alta na chegada da } \\
\text { SRPA, bem como } \\
\text { demorava menos tempo } \\
\text { para atingir a temperatura } \\
36,5^{\circ} \text { (normotermia) na } \\
\text { SRPA em comparação com } \\
\text { os outros métodos. A } \\
\text { tecnologia reflexiva } \\
\text { mostrou-se menos eficiente } \\
\text { que os cobertores de } \\
\text { algodão. }\end{array}$ & $\begin{array}{l}\text { Apesar do método que se } \\
\text { mostrou mais efetivo ser } \\
\text { mais caro, ele reduz o } \\
\text { tempo do paciente na } \\
\text { SRPA (custo de } \\
\text { aproximadamente } \$ 20 \text { por } \\
\text { cada } 15 \text { minutos) e reduz o } \\
\text { custo do tratamento das } \\
\text { complicações associadas à } \\
\text { hipotermia no período } \\
\text { perioperatório. }\end{array}$ \\
\hline
\end{tabular}

No estudo acima testou-se a aplicação de dois cobertores de algodão (controle), comparados ao método de insulação refletiva (grupo experimental 1) e o cobertor de ar aquecido artificialmente (método experimental 2), conclui-se que o grupo que recebeu o método do cobertor de ar aquecido apresentou temperatura mais alta na chegada da Sala de Recuperação Pós Anestésica (SRPA), bem como demorava menos tempo para atingir a temperatura

\section{Caracterização da Amostra}

Dos artigos encontrados analisamos três artigos que atendiam aos critérios de inclusão propostos no presente estudo. Os autores de duas das publicações analisadas eram predominantemente médicos e apenas um dos estudos foi conduzido por enfermeiras. Dentre estes artigos, um foi realizado em instituição hospitalar e dois vinculados a Universidades. Foram conduzidos nos seguintes países: Singapura, Japão e Estados Unidos. Os periódicos analisados eram dois médicos e um de enfermagem. Dois estudos foram publicados em 2003 e um estudo foi publicado em 1997.

O delineamento utilizado em dois dos estudos foi o experimental, sendo do tipo ensaio clínico controlado randomizado, desta forma de acordo com o nível de evidência proposto por STETLER et al (1998) foram classificados como nível 2 e um deles do tipo quase experimental (porque não inclui grupo controle) sendo classificado como nível 3.

Apresentamos a seguir a análise dos estudos investigados de forma individual, de acordo, com o detalhamento metodológico, intervenção realizada, resultados obtidos e conclusões.

NEGISHI, C. Resistive heating and forced-air warming are comparably effective. Anesthesia \& Analgesia, v.96, n.6, p. 1683-7, june 2003. 
POVEDA, Vanessa de Brito; PICCOLI, Marister; GALVÃO, Cristinha Maria; SAWADA, Namie Okino. MÉTODOS DE PREVENÇÃo E REAQUECIMENTO DO PACIENTE PARA O PERIOPERATÓRIO. Revista Eletrônica de Enfermagem, v. $07, n .03, p .266$ - $272,2005$. Disponível em http://www.fen.ufg.br/Revista/revista7 3/original 02.htm

\begin{tabular}{|c|c|c|c|}
\hline $\begin{array}{l}\text { Detalhamento } \\
\text { Metodológico }\end{array}$ & Intervenção Estudada & Resultados & $\begin{array}{l}\text { Recomendações/ } \\
\text { Conclusões }\end{array}$ \\
\hline $\begin{array}{lr}\text { Estudo quase experimental } \\
24 \text { pacientes submetidos a } \\
\text { cirurgias } & \text { eletivas } \\
\text { abdominais } & \text { abertas, } \\
\text { divididos em três grupos } \\
\text { por meio de seleção } \\
\text { randômica. }\end{array}$ & $\begin{array}{l}\text { GI - colchão de água } \\
\text { aquecida a } 42^{\circ} \\
\text { GIl - cobertor de ar } \\
\text { aquecido artificialmente } \\
\text { GllI - cobertor resistive } \\
\text { heating }\end{array}$ & $\begin{array}{l}\text { Em cirurgias abdominais } \\
\text { abertas o resistive heating } \\
\text { mantêm a temperatura } \\
\text { corporal tão eficientemente } \\
\text { quanto o cobertor de ar } \\
\text { aquecido. }\end{array}$ & $\begin{array}{l}\text { Apoiado na literatura e em } \\
\text { seus resultados ele diz que } \\
\text { o colchão de água } \\
\text { aquecida artificialmente é } \\
\text { ineficiente em manter a } \\
\text { temperatura corporal dos } \\
\text { pacientes. Os grupos } \\
\text { submetidos ao cobertor de } \\
\text { ar aquecido artificialmente } \\
\text { e ao resistive heating são } \\
\text { comparáveis quanto a sua } \\
\text { efetividade e mais efetivos } \\
\text { que o colchão de água }\end{array}$ \\
\hline
\end{tabular}

No estudo desenvolvido por NEGISHI (2003) procuraram comparar as mudanças da temperatura corporal durante cirurgias abdominais abertas utilizando-se o colchão de água, cobertor de ar aquecido artificialmente e resistive heating, por meio de um delineamento quase experimental, já que o estudo em questão não estabeleceu grupo controle. Verificou que em cirurgias abdominais abertas o resistive heating mantêm a temperatura corporal tão eficientemente quanto o cobertor de ar aquecido, e ainda, apoiado na literatura e em seus resultados ele diz que o colchão de água aquecida artificialmente é ineficiente em manter a temperatura corporal dos pacientes, sendo que, os grupos que receberam o método do cobertor de ar aquecido artificialmente e o resistive heating são comparáveis quanto a sua efetividade e mais efetivos que o colchão de água.

HERSHEY, J.; VALENCIANO, C.; BOOKBINDER, M. Comparison of three rewarming methods in a Postanesthesia Care Unit. AORN J, v. 65, n.3, p. 597-601, mar 1997.

\begin{tabular}{|c|c|c|c|}
\hline $\begin{array}{l}\text { Detalhamento } \\
\text { Metodológico }\end{array}$ & Intervenção Estudada & Resultados & \begin{tabular}{|l|} 
Recomendaçõesl \\
Conclusões
\end{tabular} \\
\hline $\begin{array}{l}\text { Ensaio clínico randomizado } \\
\text { Iniciou-se o estudo com } \\
144 \text { pacientes } \\
\text { permanecendo ao final } \\
\text { com } 140 \text { por perdas de } \\
\text { dados durante o estudo, } \\
\text { estes foram randomizados } \\
\text { em três grupos. }\end{array}$ & $\begin{array}{l}\text { controle - dois cobertores } \\
\text { aquecidos e a colcha da } \\
\text { cama do hospital } \\
\text { GI - o método padrão do } \\
\text { hospital mais o cobertor de } \\
\text { alumínio reflexivo } \\
\text { GIl - o método de } \\
\text { aquecimento padrão mais } \\
\text { o cobertor de alumino } \\
\text { reflexivo mais reflective } \\
\text { head covering (touca } \\
\text { reflexiva). }\end{array}$ & $\begin{array}{l}\text { Não existiram diferenças } \\
\text { estatisticamente } \\
\text { significantes entre os } \\
\text { grupos }\end{array}$ & $\begin{array}{l}\text { Apesar do estudo não ter } \\
\text { mostrado diferenças entre } \\
\text { os grupos ele serviu para a } \\
\text { instituição ficar mais atenta } \\
\text { quanto à hipotermia, } \\
\text { utilizando após o estudo } \\
\text { métodos de aquecimento } \\
\text { no trans- operatório e } \\
\text { SRPA }\end{array}$ \\
\hline
\end{tabular}

HERSHEY et al, (1997) desenvolveram um estudo onde procuraram testar a efetividade de 3 métodos de aquecimento para a recuperação da temperatura corporal dos pacientes na SRPA, sendo eles, como controle, dois cobertores aquecidos e a colcha da cama do hospital, grupo experimental 1, o método padrão do hospital mais o cobertor de alumínio reflexivo e grupo experimental 2, o método de aquecimento padrão mais o cobertor de alumino reflexivo mais reflective head covering (touca reflexiva?), seus resultados demonstraram não haverem diferenças estatisticamente significantes entre os grupos.

\section{CONCLUSÃO}

De acordo com os estudos analisados podemos concluir que o melhor método de aquecimento para os pacientes no período perioperatório, especialmente no pós-operatório imediato, é o cobertor de ar aquecido, outro método comparável seria o resistive heating.

Métodos mais conhecidos e utilizados em nossa realidade não demonstraram efetividade comparável aos métodos citados acima, como o cobertor de algodão e o colchão de água aquecida, vale lembrar também, os riscos associados ao colchão de água aquecida como queimaduras, por exemplo.

Assim, apesar do custo elevado destas tecnologias devemos ressaltar a importância da prevenção da hipotermia como uma medida efetiva na redução de custos, além dos efeitos deletérios causados por ela na recuperação pós- operatória destes pacientes. 
POVEDA, Vanessa de Brito; PICCOLI, Marister; GALVÃO, Cristinha Maria; SAWADA, Namie Okino. MÉTODOS DE PREVENÇÃo E REAQUECIMENTO DO PACIENTE PARA O PERIOPERATÓRIO. Revista Eletrônica de Enfermagem, v. $07, \mathrm{n} .03, \mathrm{p} .266$ - $272,2005$. Disponível em http://www.fen.ufg.br/Revista/revista7_3/original_02.htm

Este estudo, apesar do número reduzido de estudos localizados nos despertou para a continuidade do mesmo em decorrência da relevância do assunto para a enfermagem perioperatória, e também da possibilidade da divulgação de técnicas na prevenção e reabilitação da normotermia para a enfermagem. Outro aspecto que consideramos relevante e também foi despertado a partir da realização deste trabalho foi à identificação de lacunas, como por exemplo, os parâmetros de normotermia, hipotermia leve e hipotermia utilizados pelos autores e que também merece futuras investigações.

\section{REFERÊNCIAS BIBLIOGRÁFICAS}

AMERICAN SOCIETY OF PERIANESTHESIA NURSES. Clinical guideline for the prevention of unplanned perioperative hypothermia. Journal of PeriAnesthesia Nursing, v.16, n.5, p. 305-314. 2001.

BORMS, S. et al. Bair-hugger forced-air warming maintains normothermia more effectively than thermolite insulation. Journal of Clinical Anesthesia, n.6, p.303-307, 1994.

CILISKA, D.; CULLUM, N.; MARKS, S. Evaluation of systematic reviews of treatment or prevention interventions. Evidence-Based Nursing, v. 4, n. 4, p. 100-04, 2001.

DOUFAS, A.G. Consequences of inadvertent perioperative hypothermia. Best Practice \& Research Clinical Anaesthesiology, v. 17, n.4, p.535-549, 2003.

ESTABROOKS, C.A. Will evidence-based nursing practice make practice perfects? Canadian Journal of Nursing Research, v. 30, n.1, p. 15-36, 1998.

GALVÃ̃, C. M. A prática baseada em evidências: uma contribuição para a melhoria da assistência de enfermagem perioperatória. Ribeirão Preto, 2002. 114p. Tese (Livre-docência). Escola de Enfermagem de Ribeirão Preto, Universidade de São Paulo.

GALVÃO, C. M.; SAWADA, N.O. TREVIZAN, M.A. Revisão sistemática: recurso que proporciona a incorporação das evidências na prática da enfermagem. Rev. Latino-am Enfermagem, v. 12, n.3, p.549-56, 2004.

GARANHANI, M.L.; KEMMER, L.F.; RODRIGUES, A.I. Avaliação de métodos de aquecimento aplicados ao paciente hipotérmico na sala de recuperação pósanestésica - S.R.P.A. Revista Paulista de Enfermagem, v.9, n.3, p.88-96, 1990.

GUYTON, A.C.; HALL, J.E. Temperatura corporal, regulação térmica e febre. In: GUYTON, A.C \& HALL, J.E. Tratado de fisiologia médica. Rio de Janeiro: Guanabara-Koogan, 2002. Cap.73, p.769-779.

HAMER, S. Evidence-based practice. In: HAMER, S.; COLLINSON, G. Achieving evidence-based practice: a handbook for practitioners. London: Baillière Tindall, 1999. Chap. 1, p. 3-12.

HERSHEY, J.; VALENCIANO, C.; BOOKBINDER, M. Comparison of three rewarming methods in a Postanesthesia Care Unit. AORN J, v. 65, n.3, p. 597601, 1997.
LESLIE, K.; SESSLER, D.I. Perioperative hypothermia in the high-risk surgical patient. Best Practice \& Research Clinical Anaesthesiology, v. 17, n.4, p.485498, 2003.

MOLONEY, R.; MAGGS, C. A systematic review of the relationships between written manual nursing care planning, record keeping and patient outcomes. J. Adv. Nursing, v. 30, n. 1, p. 51-7, 1999.

MANNING, B.; STOLLERMAN, G.H. Hypothermia in the elderly. Hospital Practice: Office Edition, v.28, n.5, p.53-70, 1993.

NEGISHI, C. Resistive heating and forced-air warming are comparably effective. Anesthesia \& Analgesia, v.96, n.6, p. 1683-7, 2003.

NG, S.F. et al. A comparative study of three warming interventions to determine the most effective in maintaining perioperative normothermia. Anesthesia \& Analgesia, v.96, n.1, p. 171-6, 2003.

PETERSON, C. Table overhang; hypothermia; separating sponges; skin lacerations when scrubbing; wound classification; forced air warming. AORN J, v. 73, n.1, p. 123-131, 2003.

POLIT, D.F.; BECK, C.T.; HUNGLER, B.P. Fundamentos de Pesquisa em Enfermagem: métodos, avaliação e utilização. 5. ed. Porto Alegre: Artmed, 2004.

STETLER, C.B. et al. Utilization - focused integrative reviews in a nursing service. Applied Nursing Research, v.11, n.4, p.195-206,1998.

STEVENS, D.; JOHNSON, M.; LANGDON, R. Comparison of two warming interventions in surgical patients with mild and moderate hypothermia. International Journal of Nursing Practice, v. 6, n.5, p.268-275, 2000.

TRAMONTINI, C.C. Controle da hipotermia de pacientes cirúrgicos idosos no intra-operatório: avaliação de duas intervenções de enfermagem. São Paulo, 2000. 107p. Dissertação (Mestrado). Escola de Enfermagem, Universidade de São Paulo.

URSI, E.S. Prevenção de lesões de pele no perioperatório: revisão integrativa da literatura. Ribeirão Preto, 2005. 128 p. Dissertação (Mestrado). Escola de Enfermagem de Ribeirão Preto, Universidade de São Paulo.

Texto recebido em 25/10/2005.

Publicação aprovada em 10/12/2006. 